\title{
Avaliação de diferentes métodos de preservação do fungo Phoma Dimorpha
}

\author{
José Roberto Chaves Neto ${ }^{1}$, Marcio Antonio Mazutti ${ }^{1}$, Giovani Leone Zabot ${ }^{2}$, Marcus Vinícius Tres ${ }^{2}$ \\ ${ }^{1}$ Universidade Federal de Santa Maria - UFSM, Departamento de Engenharia Química, Santa Maria, RS. ${ }^{2}$ Universidade \\ Federal de Santa Maria - UFSM, Laboratório de Engenharia de Processos Agroindustriais, Cachoeira do Sul, RS. E-mail: \\ jose.chavesneto@gmail.com
}

\begin{abstract}
Resumo
Frente a necessidade de preservação e a manutenção de materiais biológicos, dentre eles fungos com potencial para controle biológico, para o desenvolvimento biotecnológico e científico, que vêm ganhando destaque no cenário mundial. Sendo necessário a adequação de métodos de preservação que além de garantia a sobrevivência destes microrganismos permitam a conservação de suas características morfológicas, fisiológicas e genéticas, no entanto, não existe um método ideal ou universal para a conservação de materiais biológicos. Diante desta necessidade o presente trabalho teve como objetivo de avaliar a eficácia e viabilidade de três métodos de preservação de isolados do fungo Phoma dimorpha (repicagens periódicas, Castellani e fragmentos de papel-filtro), em dois períodos de avaliação, seis e doze meses após o armazenamento. Estudou-se a eficácia e viabilidade, através do crescimento micelial do fungo em meio de cultivo contendo batata-dextrose-ágar. Houve variabilidade entre os métodos de preservação do isolado do fungo $P$. dimorpha para o crescimento micelial, eficácia do método e índice de velocidade do crescimento micelial, nos dois períodos de avaliação, após seis e doze meses de armazenamento. $O$ método de preservação em fragmento de papel filtro mostrou-se como o mais eficaz na preservação do isolado do fungo $P$. dimorpha nos dois períodos de avaliação, após seis e doze meses de armazenamento, sendo ideal para obter o maior o crescimento micelial, eficácia do método e índice de velocidade do crescimento micelial.
\end{abstract}

Palavras-chave: crescimento micelial; repicagens periódicas; Castellani; fragmentos de papel-filtro.

\section{Evaluation of different methods of preservation of Phoma dimorpha fungus}

\begin{abstract}
Facing the need for preservation and maintenance of biological materials, among them fungi with potential for biological control, for biotechnological and scientific development, which are gaining prominence in the world scenario. It is necessary to adapt preservation methods that besides guaranteeing the survival of these microorganisms allow the conservation of their morphological, physiological and genetic characteristics, however, there is no ideal or universal method for the conservation of biological materials. In view of this need, the present work had the objective of evaluating the efficacy and feasibility of three methods for the preservation of Phoma dimorpha (periodic transfer, Castellani and filter paper fragments) isolates in two evaluation periods, six and twelve months after the storage. Efficacy and viability were studied by mycelial growth of the fungus in a culture medium containing potato-dextrose-agar. There was variability between the preservation methods of the $P$. dimorpha fungus isolate for mycelial growth, method efficacy and mycelial growth rate index, in the two evaluation periods, after six and twelve months of storage. The filter paper fragment preservation method was the most effective in preserving the $P$. dimorpha fungus isolate in the two evaluation periods, after six and twelve months of storage, being ideal to obtain the highest mycelial growth, efficacy of the method and mycelial growth rate index.
\end{abstract}

Keywords: mycelial growth; periodic transfer; Castellani method; fragments of qualitative filter paper. 


\section{Introdução}

Os microrganismos são tidos como uma grande fonte de compostos de alto valor biológico que podem ser utilizados em diversas áreas, porém pouco investigado. Dentre estes compostos produzidos pelos microrganismos, destacam-se vitaminas, antibióticos, álcool, enzimas, biossurfactantes, fitotoxinas, entre outros (CAMEOTRA, 2007). Os fungos são os mais investigados, pois diversos espécies possuem a capacidade de produzir uma grande variedade de metabólitos secundários quimicamente novos e com expressiva atividade biológica (HINZ et al., 2014; VILLAVERDE et al., 2014; WIJERATNE et al., 2013).

Os fungos do gênero Phoma estão entre os mais promissores produtores de metabólitos secundários, possuindo grande potencial para o controle biológico, que podem ser explorados para a produção de produtos fitossanitários naturais para agricultura sustentável (KIM et al., 2012; RAl et al., 2009). Diversas pesquisas vêm mostrando que algumas espécies deste gênero apresentam elevado potencial para produção de metabólitos secundários com atividade herbicida (CIMMINO et al., 2013; EVIDENTE et al., 2015; ARORA et al., 2016; BRUN et al., 2016; KLAIC et al., 2017; TODERO et al., 2018a,b). Além disso, algumas pesquisas também mostram que as moléculas bioativas produzidas por estes fungos possuem atividade antibacterianas e antifúngicas (KOYAMA et al., 2005; YANO et al., 2007; SANTIAGO et al., 2012, HUSSAIN et al., 2014).

Segundo Guimarães (2011), o isolamento e melhoramento de fungos são processos longos e de elevado custo, sendo necessário a seleção e adequação de técnicas de preservação, visto que está medida permitirá a realização de pesquisas, tanto básicas como aplicadas, a qualquer tempo. Atualmente no cenário mundial a preservação de materiais biológicos vem ganhando destaque, devido à necessidade destes para o desenvolvimento biotecnológico e científico. A utilização de métodos de preservação tem como proposito permitir a sobrevivência do microrganismo de interesse, garantir a manutenção das características originais da cultura: morfológicas, fisiológicas (esporulação e patogenicidade) e genéticas, excluindo mutações e contaminação indesejável, mesmo após longos períodos (BELOTI et al., 2017).

Para a escolha do método de preservação é necessário levar em consideração alguns fatores, como as características do material biológico em estudo, assim como as vantagens e desvantagens dos métodos disponíveis, dos custos de manutenção, e da disponibilidade de equipamentos para tal procedimento (NAKASONE et al., 2004). Desse modo, a determinação do melhor método de preservar fungos, com a utilização de técnicas simples e eficientes, é de grande interesse para laboratórios de microbiologia. Além de permite o desenvolvimento de estudos capazes de superar barreiras cronológicas e geográficas e, por conseguinte, elucidar as particularidades dos diferentes materiais biológicos (TERAMOTO et al., 2011).

Os métodos de preservação de microrganismos mais utilizados são temperaturas baixas ou congelamento, nitrogênio líquido, repicagens periódicas ou repique continuo, Castellani ou água destilada, liofilização e óleo mineral, além destes, outros métodos, como o de sílica-gel, fragmento de papel filtro, grãos de cereais esterilizados, solo e turfa esterilizados, também podem ser empregados (BUENO, 2006; APARECIDO et al., 2012). Segundo Finatti e Aparecido (2009), os métodos mais utilizados em institutos de pesquisas, como o Instituto Biológico de Campinas, para preservação de fitopatógenos fúngicos são 0 método de repicagens periódicas, óleo mineral, Castellani e liofilização. Estes autores relatam que diversas pesquisas vêm mostrando bons resultados, para longos períodos de preservação.

Diante disso, o objetivo deste trabalho foi avaliar a eficácia e viabilidade de três métodos de preservação de isolado do fungo Phoma dimorpha (repicagens periódicas, Castellani e fragmentos de papel-filtro), em dois períodos de avaliação, seis e doze meses após o armazenamento.

\section{Material e métodos}

O ensaio foi conduzido no laboratório Biotec Factory do Departamento de Engenharia Química da Universidade Federal de Santa Maria, Santa Maria, RS.

$O$ fungo submetido aos diferentes métodos de preservação foi a espécie Phoma dimorpha Aveskamp, Gruyter \& Verkley. As cepas deste fungo foram provenientes da coleção do laboratório o Biotec Factory do Departamento de Engenharia Química da Universidade Federal de Santa Maria, Santa Maria, RS.

Os ensaios foram conduzidos utilizando um delineamento experimental inteiramente 
casualizado (DIC), com três tratamentos e quatro repetições. Os tratamentos foram constituídos de três métodos de preservação: repique contínuo, fragmentos de papel filtro e água destilada estéril (método de Castellani).

A preservação dos isolados de $P$. dimorpha nos três métodos foi realizada em setembro de 2017 e as avaliações de viabilidade foram realizadas em março e outubro de 2018, aos seis e dose meses após armazenamento, respectivamente, totalizando doze meses de avaliação dos métodos de preservação.

O método de repicagens periódicas ou repique continuo foi realizado de acordo com metodologia descrita por Dhingra e Sinclair (1995). Um disco de micélio do fungo foi repicado, para placas de petri (Figura 4) contendo meio BDA, em seguida seladas com parafilme, e incubadas por 10 dias, a $28^{\circ} \mathrm{C}$ na ausência de luz. Após o crescimento micelial as placas foram armazenadas em geladeira à temperatura de $4 \mathrm{a}$ $8^{\circ} \mathrm{C}$, e a cada dois meses o fungo era repicado para outras placas com meio BDA.

Para a preservação em fragmentos de papel-filtro, utilizou-se a metodologia proposta por Alfenas e Mafia (2007). Tiras de papel filtro esterilizados $(2,5 \times 0,7 \mathrm{~cm})$, foram depositados sobre meio de cultura BDA em placas de petri. Posteriormente, discos de micélio do fungo foram repicados para as placas visando proporcionar o crescimento do fungo sobre os fragmentos de papel. As placas foram incubadas por 10 dias, a $28{ }^{\circ} \mathrm{C}$ na ausência de luz. Após o crescimento micelial sobre os fragmentos de papel, os mesmos foram transferidos para placas de Petri estéreis, e permaneceram em incubadora nas mesmas condições pelo período de 10 dias para a secagem das tiras de papel contendo o micélio do fungo. Posteriormente, os fragmentos de papel foram transferidos para microtubos de $2 \mathrm{~mL}$ do tipo Eppendorf e armazenados a -15C. Foram armazenadas quatro tiras de papel com o fungo por frasco.

A preservação em água destilada estéril ou método de Castellani foi realizada de acordo com metodologia descrita por Alfenas e Mafia (2007). Para isso, inicialmente, frascos-ampola de vidro, com capacidade para $10 \mathrm{~mL}$, foram preenchidos com $4 \mathrm{~mL}$ de água destilada e vedados com rolhas de borracha, em seguida autoclavados a 121 oC por 30 minutos. Posteriormente, em câmara asséptica, foram transferidos quatro discos de micélio $(6 \mathrm{~mm}$ de diâmetro) de meio BDA provenientes de colônias em crescimento ativo do fungo $P$. dimorpha em cada frasco.

As avaliações de eficácia e viabilidade dos métodos de preservação para os isolados de $P$. dimorpha foram realizadas, em dois períodos de avaliação, aos seis e doze meses após o armazenamento, onde discos de micélio foram retirados e colocados em meio BDA para teste de eficácia e viabilidade dos métodos de preservação avaliados.

Para ambos os métodos de preservação as avaliações tiveram como base medições diárias do diâmetro das colônias, com auxílio de um paquímetro digital (Within $150 \mathrm{~mm}$ ), em eixos ortogonais (média das duas medidas diametralmente opostas) para obter-se a média do crescimento miceliano diário, até que o tratamento testemunha atingisse os bordos da placa (MAIA et al., 2011). Com base nestes dados calculou-se:

0 índice de velocidade do crescimento micelial (IVCM - mm dia ${ }^{-1}$ ), foi determinado com base na fórmula descrita por Maia et al. (2011):

$$
\text { IVCM }=\sum \frac{(D-D a)}{N}
$$

Onde: $\mathrm{D}=$ diâmetro médio atual da colônia $(\mathrm{mm})$; $\mathrm{Da}=$ diâmetro médio da colônia do dia anterior $(\mathrm{mm}) ; \mathrm{N}=$ número de horas ou dias após a inoculação.

A eficácia de cada método de preservação (EMP - \%), foi calculada a partir da adaptação da equação descrita por Abbott (1925), utilizando-se os dados de crescimento micelial:

Onde:

$$
\mathrm{EMP}=(100 \times \mathrm{CM} / 60)
$$

$\mathrm{CM}=$ Crescimento micelial médio do método de preservação;

$E M P=$ Eficácia do método de preservação.

Os dados foram submetidos aos testes de normalidade e homogeneidade dos erros, do qual foi contatado o cumprimento dos pressupostos, em seguida, os dados foram submetidos à análise de variância e na presença de significância, as variáveis qualitativas tiveram suas médias comparadas pelo teste de Tukey, ao nível de $5 \%$ de probabilidade de erro, com auxílio do programa estatístico Sisvar versão 5.1 (FERREIRA, 2014). 


\section{Resultados E Discussão}

Os resultados da análise da variância e dos valores médios de crescimento micelial, eficácia do método de preservação (EMP) e índice de velocidade do crescimento micelial (IVCM) do fungo $P$. dimorpha em função dos diferentes métodos de preservação, após seis ( 6 $\mathrm{MA})$ e doze meses de armazenamento (12MA), indicam que houve diferença significativa entre os métodos de preservação (Tabela 1). Os baixos valores de coeficiente de variação mostram que os dados observados satisfazem a premissa de normalidade.

Tabela 1. Resumo da análise de variância e valores médios de crescimento micelial (CM - mm), eficácia do método de preservação (EMP - \%) e índice de velocidade do crescimento micelial (IVCM) do fungo $P$. dimorpha em função do método de preservação, em seis (6 MA) e doze meses de armazenamento (12MA)

\begin{tabular}{cccccccc}
\hline \multirow{2}{*}{${ }^{1} \mathrm{FV}$} & & \multicolumn{6}{c}{ Quadrados médios } \\
\cline { 3 - 8 } & ${ }^{2} \mathrm{GL}$ & \multicolumn{2}{c}{$\mathrm{CM}(\mathrm{mm})$} & \multicolumn{2}{c}{$\mathrm{EMP}(\%)$} & \multicolumn{1}{c}{ IVCM (mm/dia $\left.{ }^{-1}\right)$} \\
\cline { 2 - 7 } & & $6 \mathrm{MA}$ & $12 \mathrm{MA}$ & $6 \mathrm{MA}$ & $12 \mathrm{MA}$ & $6 \mathrm{MA}$ & $12 \mathrm{MA}$ \\
\hline Método de Preservação & 2 & $70,20^{*}$ & $154,93^{*}$ & $42,83^{*}$ & $430,44^{*}$ & $84,83^{*}$ & $43,21^{*}$ \\
Erro & 9 & 16,17 & 18,37 & 3,80 & 50,11 & 0,63 & 0,89 \\
\hline Desvio Padrão & 1,98 & 6,55 & 3,30 & 10,92 & 3,93 & 2,93 \\
Coeficiente de variação (\%) & 12,09 & 11,99 & 2,85 & 11,99 & 5,02 & 7,85 \\
\hline
\end{tabular}

${ }^{(1)}$ Fonte de variação, ${ }^{(2)}$ Grau de liberdade, *significativo a $5 \%$ e ${ }^{(\mathrm{NS})}$ não significativo.

Ao analisar os diferentes métodos de preservação do fungo $P$. dimorpha, constatou-se que todos diferem entre si $(p \leq 0,05)$, nos dois períodos de avaliação, aos seis e doze meses após o armazenamento. $O$ método de preservação em fragmentos de papel-filtro foi 0 que proporcionou os maiores diâmetros de crescimento micelial, com valores médios superiores a $43 \mathrm{~mm}$, nos dois períodos de avaliação, aos seis e doze meses após o armazenamento, diferindo estatisticamente dos demais métodos (Figura 1A, 2).

Quanto a porcentagem de eficácia dos métodos de preservação do fungo $P$. dimorpha
(Figura 1B), constatou-se diferença significativa entre os métodos de preservação avaliados neste trabalho. Sendo a maior porcentagem de eficácia observada no método de preservação em fragmento de papel-filtro nos dois períodos de avaliação, com porcentagem média superior a 70 e 65, aos seis e doze meses após o armazenamento, respectivamente. Com base neste resultado confirma-se a eficácia do método de preservação em fragmento de papel-filtro para o fungo $P$. dimorpha. 
Figura 1. Médias do crescimento micelial e porcentagem de eficácia dos métodos de preservação dos diferentes métodos de preservação do fungo $P$. dimorpha, em dois períodos de avaliação, aos seis e doze meses após o armazenamento. *Médias seguidas de mesma letra, não diferem estatisticamente entre si pelo teste de Tukey a $5 \%$ de probabilidade.
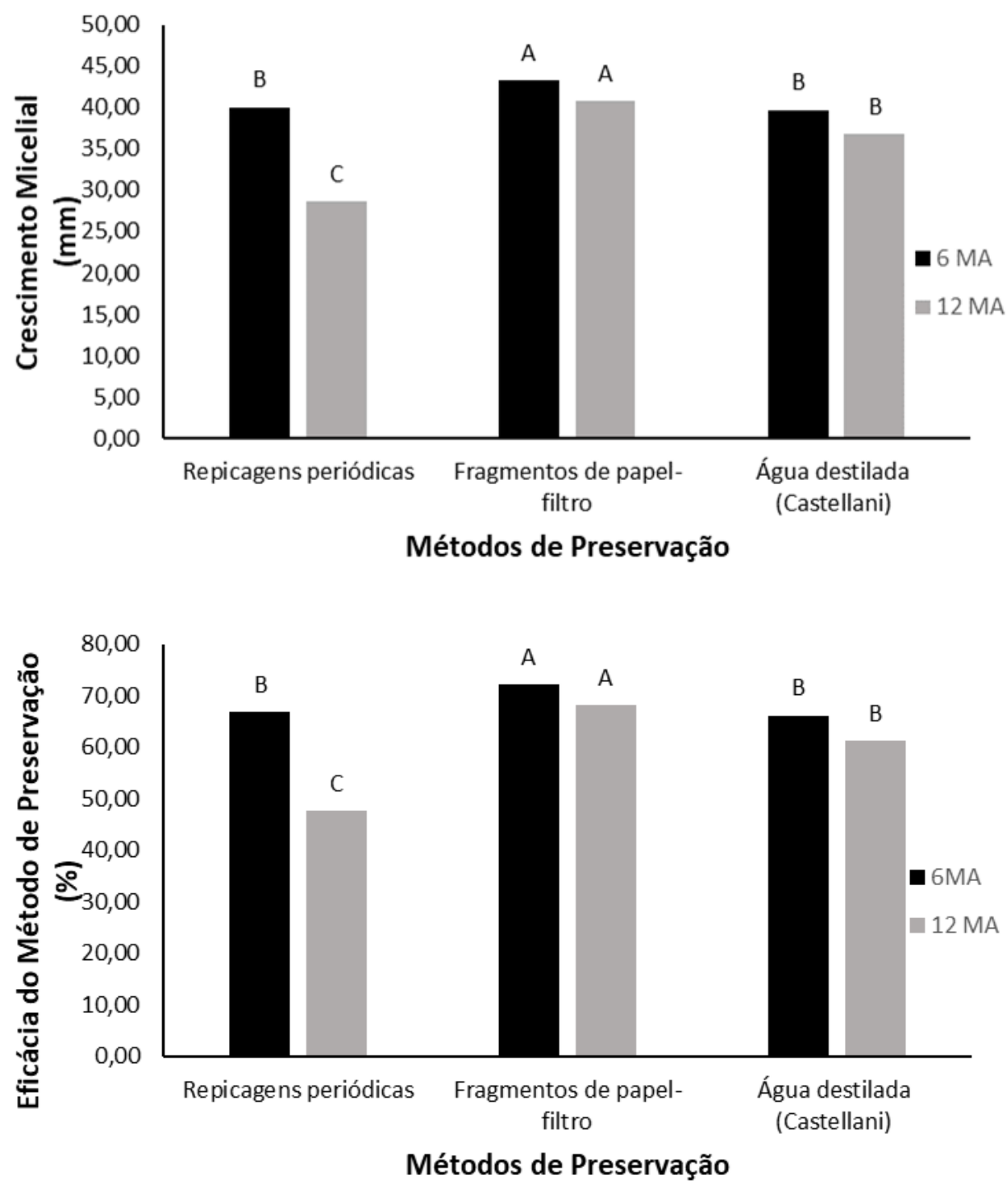

Os resultados obtidos neste estudo destacam que o método de fragmento de papel filtro foi o método de preservação que permitiu maior crescimento micelial, para os isolados do fungo $P$. dimorpha, seguido do repique contínuo e Castellani. No entanto, este resultado não pode ser generalizado para outros fungos, visto que, em virtude da grande biodiversidade destes microrganismos, de acordo Girão et al. (2004) não existe uma técnica padrão que seja capaz de preservá-los de forma adequada. Existem diversos métodos para preservação de fungos, porém para a escolha do método de manutenção mais adequado devem-se levar em consideração as vantagens e desvantagens de cada técnica disponível e principalmente as características do agente em estudo (SOLA et al., 2012). 
Figura 2. Recuperação de isolados de $P$. dimorpha preservados pelos métodos de repicagens periódicas (RP), fragmentos de papel-filtro (PF) e água destilada (AD), em dois períodos de avaliação, aos seis e doze meses após o armazenamento.
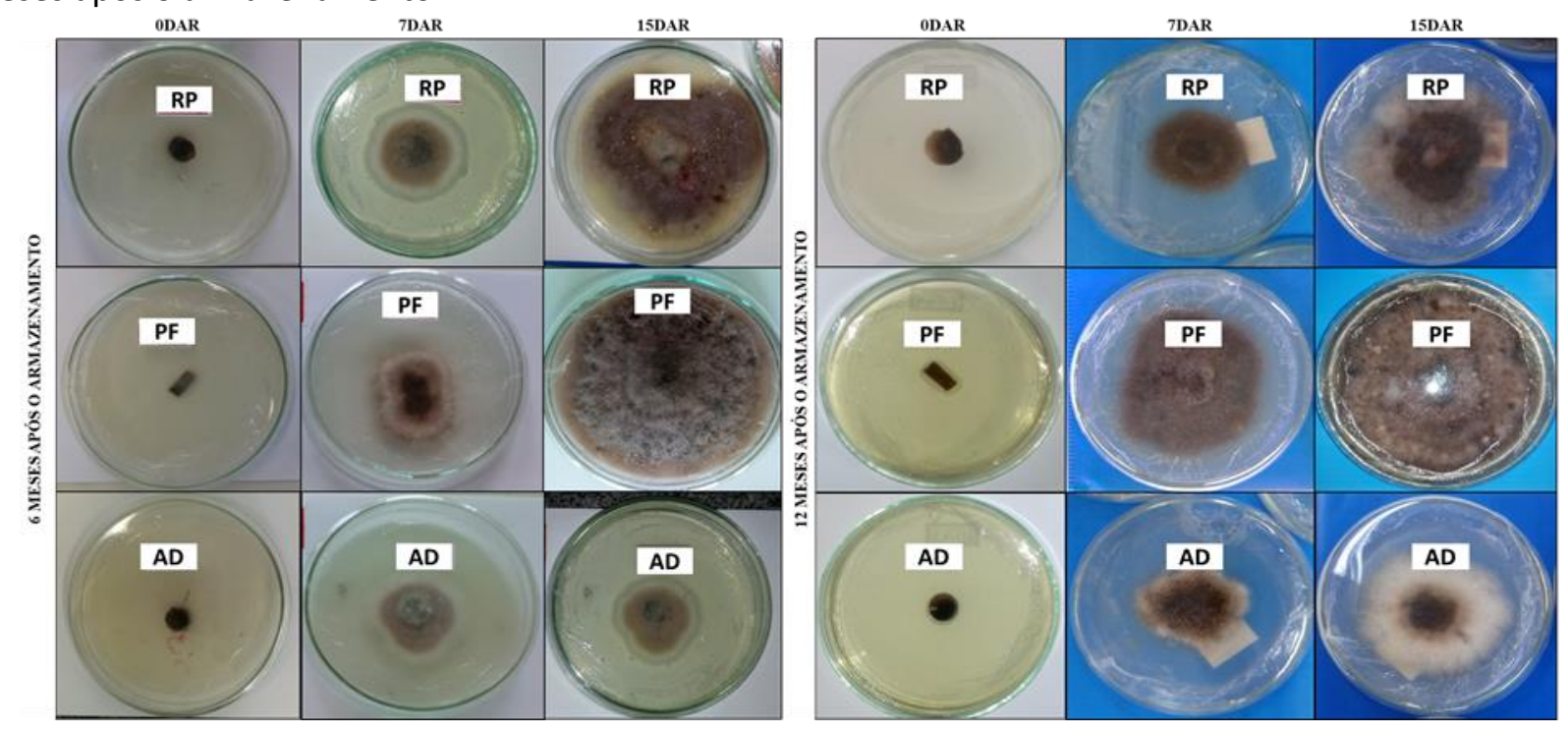

A eficácia do método de preservação em fragmento de papel-filtro também foi descrita por Fong et al. (2000), os quais descrevem que o método de preservação em fragmento de papelfiltro se mostrou muito eficaz para a preservação de $F$. oxysporum a $-19{ }^{\circ} \mathrm{C}$, mantendo-se viáveis após quatro anos de armazenamento, e todas as culturas foram recuperadas sem a presença de ácaros. Neste método de preservação ocorre a remoção de água das células fúngicas, o que diminui ou suspende $\mathrm{o}$ metabolismo, aumentando o período de armazenamento na geladeira prolongando a longevidade das culturas (DHINGRA; SINCLAIR, 1995; FONG et al., 2000).

A preservação em fragmento de papel filtro, apresenta baixo custo, não necessita de equipamentos sofisticados, além de estabilidade elevada e a não ocorrência de ácaros em função de ser mantido a baixas temperaturas (ANJOS et al., 2012). De acordo com estudo desenvolvido por Aparecido et al. (2012), isolados de Phoma spp. mostraram-se viáveis após preservação de dois meses, pelo método de congelamento de estruturas a $-80^{\circ} \mathrm{C}$.

Com base na figura 1 , os métodos de preservação repicagens periódicas e Castellani apresentaram os menores valores crescimento micelial, sendo semelhantes quanto ao crescimento micelial no primeiro período de avaliação (6MA) e diferiram no segundo aos doze meses após o armazenamento, com valores médios de crescimento micelial variando de 28 a $40 \mathrm{~mm}$.

De acordo com Aparecido et al. (2007) comparando viabilidade de culturas fúngicas preservadas pelos métodos de Castellani e liofilização com base nos dados de crescimento micelial, constataram que o método de Castellani (água destilada) foi o mais vantajoso para manter, em laboratório, diferentes gêneros e espécies de fungos. Comparando os métodos de repicagens periódicas, método de Castellani ou água destilada e liofilização para manutenção de 35 culturas fúngicas fitopatogênicas foi possível verificar que o método o método de Castellani ou água destilada foi o mais adequado, por ser o mais eficiente na manutenção da viabilidade e esporulação, apresentou baixa taxa de mutações e de contaminação (PIRES et al., 2012).

Passador et al. (2010), avaliando a manutenção da viabilidade dos Verticillium dahliae e Fusarium oxysporum, pelos métodos de Castellani (água destilada) e repicagens periódicas, constataram que em ambos os métodos o fungo $V$. dahliae permaneceu viável por seis anos. Avaliando a eficiência de três métodos de preservação de isolados, durante seis meses de armazenamento Teramoto et al., (2011), constataram que o método de repicagem periódica foi o método de preservação que permitiu maior crescimento micelial, para todos os isolados, seguido do Castellani e do óleo mineral.

A longo prazo as culturas mantidas por meio de repicagens periódicas podem apresentar contaminação por ácaros ou outros 
microrganismos, além de ocorrer a secagem do meio. Uma outra desvantagem é que a morfologia e fisiologia de um fungo cultivado podem mudar ao longo do tempo, como a capacidade de esporular ou de infectar um hospedeiro, podem ser perdidas após repiques contínuos por longos períodos (DHINGRA; SINCLAIR, 1995; NAKASONE et al., 2004).

De acordo com Green (2008) e Costa et al. (2009), ao longo dos anos vários estudos vêm sendo realizados com o objetivo de desenvolver e aplicar protocolos, no entanto, a grande maioria dos métodos não se aplicado a todos os microrganismos, devido as caraterísticas peculiaridades do microrganismo de interesse e também as características das técnicas (equipamentos e materiais). Estes autores alertam para a necessidade da conjunção de dois ou mais métodos, a fim de se garantir a melhor recuperação dos microrganismos.

$O$ índice de velocidade média de crescimento micelial (IVCM) do fungo $P$. dimorpha sofreu influência dos métodos de preservação, nos dois períodos de avaliação, aos seis e doze meses após o armazenamento (Figura 3). O maior IVCM foi observado no método de preservação em fragmento de papel-filtro, diferindo estatisticamente dos demais métodos, nos dois períodos de avaliação, com médias de 20,39 e $15,23 \mathrm{~mm} / \mathrm{dia}^{-1}$, aos seis e doze meses após o armazenamento, respectivamente. O método de preservação em fragmento de papel filtro, foi o que obteve o maior índice, $21,92 \mathrm{~mm}$ $\mathrm{dia}^{-1}$ (Figura 3).

Qiangqiang et al. (1998) comparou a viabilidade de setenta e oito isolados após doze anos, utilizando os métodos de liofilização e Castellani na preservação dos mesmos obtendo como viáveis $89,7 \%$ dos isolados preservados pelo método de Castellani e $87,2 \%$ dos isolados preservados pelo método de liofilização.

De acordo com Guimarães (2011), o repique contínuo, também chamado de subcultura ou repicagem periódica, é uma das técnicas de conservação mais antigas e amplamente utilizada, por ser um método simples e baixo custo, que permite a manutenção da grande maioria das espécies de fúngicas. No entanto, este método é uma boa opção para pequenas coleções de culturas em constante utilização por períodos curtos (menos de 1 ano).

Figura 3. Médias do índice de velocidade de crescimento micelial do fungo $P$. dimorpha nos diferentes métodos de preservação, em seis e doze meses de armazenamento. *Médias seguidas de mesma letra, não diferem estatisticamente entre si pelo teste de Tukey a $5 \%$ de probabilidade.

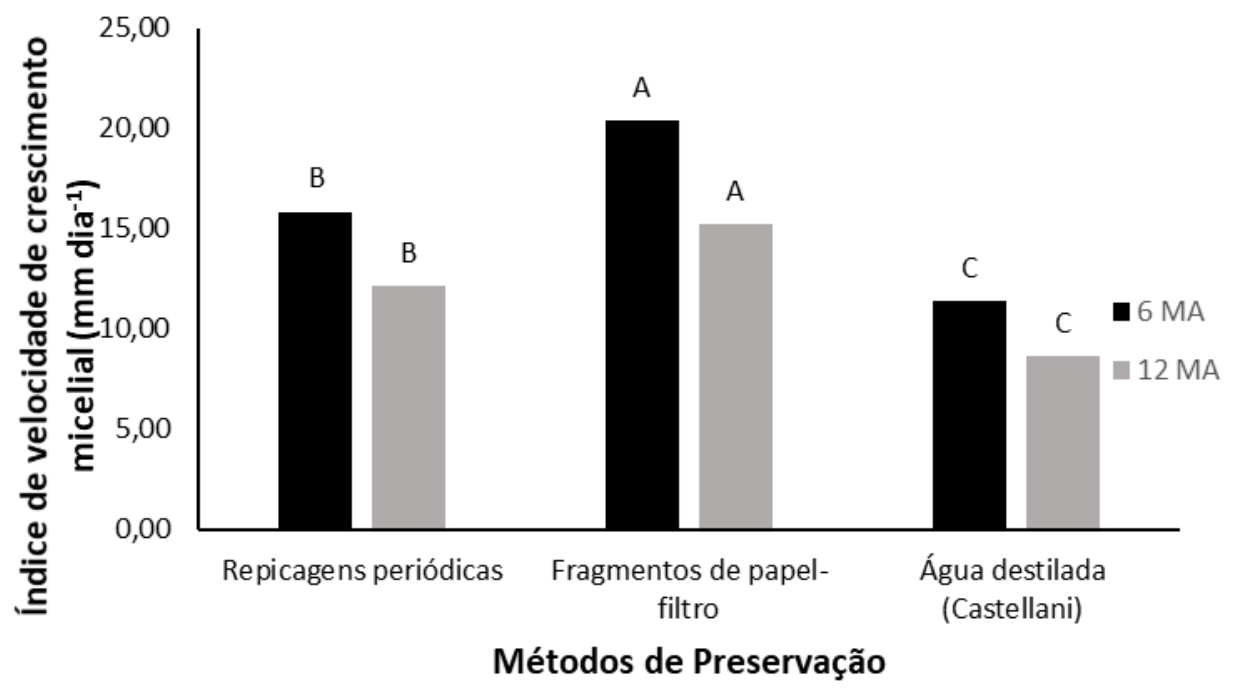

\section{Conclusões}

Houve variabilidade entre os métodos de preservação do isolado do fungo $P$. dimorpha para o crescimento micelial, eficácia do método e índice de velocidade do crescimento micelial, nos dois períodos de avaliação, após seis e doze meses de armazenamento.
O método de preservação em fragmento de papel filtro mostrou-se como o mais eficaz na preservação do isolado do fungo $P$. dimorpha nos dois períodos de avaliação, após seis e doze meses de armazenamento, sendo ideal para obtenção do maior crescimento micelial, eficácia 
do método e índice de velocidade do crescimento micelial.

\section{Agradecimentos}

Os autores agradecem à Coordenação de Aperfeiçoamento de Pessoal de Nível Superior (CAPES), ao Conselho Nacional de Desenvolvimento Científico e Tecnológico (CNPq) e à Fundação de Amparo à Pesquisa do Estado do Rio Grande do Sul (FAPERGS) pelo apoio financeiro e pela bolsa ao primeiro autor.

\section{Referências}

ABBOTT, W. S. A method of computing the effectiveness of an insecticide. Journal of Economic Entomology, v.18, n.2, p. 265-267, 1925. https://doi.org/10.1093/jee/18.2.265a

ALFENAS, A. C.; MAFIA, R. G. Métodos em fitopatologia. 2. ed. Viçosa: UFV, 2007. $516 \mathrm{p}$.

ANJOS, T. V.; TEBALDI, N. D.; FAGIAN, C. C. Coleção preservação de culturas de bactérias fitopatogênicas. Horizonte Científico, v.6, n.1, p. 1-24, 2012.

APARECIDO, C. C.; HUANG; C. T. M.; PASSADOR, M. M.; FINATTI, D.; FIGUEIREDO, M. B. Divulgação técnica: avaliação da viabilidade de culturas fúngicas preservadas pelos métodos de Castellani (água destilada) e liofilização. Biológico, v.69, n.1, p. 5-8, 2007.

APARECIDO, C. C.; PIRES, G. C. C.; FINATTI, D.; CAMILO, C. M. Preservação de micro-organismos a -80 C. Biológico, v.74, n.1, p. 23-29, 2012.

ARORA, P.; WANI, Z. A.; NALLI, Y.; ALI, A.; RIYAZUL-HASSAN, S. Antimicrobial potential of Thiodiketopiperazine derivatives produced by Phoma sp., an endophyte of Glycyrrhiza Glabra Linn. Microbial Ecology, v.72, n.4, p. 802-812, 2016. http://dx.doi.org/10.1007/s00248-016$\underline{0805-x}$

BELOTI, I. F.; JULIATTI, B. C. M.; JULIATTI, F. C. Evaluation of the gelatin technique for the preservation of phytopathogenic fungi. Bioscience Journal, v.33, n.4, p. 923-932, 2017. http://dx.doi.org/10.14393/BJ-v33n4a2017$\underline{37116}$

BRUN, T.; RABUSKE J. E.; TODERO, I.; ALMEIDA, T. C.; JUNIOR J. J. D.; ARIOTTI, G.; CONFORTIN, T.;
ARNEMANN, J. A.; KUHN, R. C.; GUEDES, J. V. C.; MAZUTTI, M. A. Production of bioherbicide by Phoma sp. in a stirred-tank bioreactor. 3 Biotech, v.6, n.2, p. 230-239, 2016.

http://dx.doi.org/10.1007/s13205-016-0557-9

CAMEOTRA, S. S. Preservation of microorganisms as deposits for patent application. Biochemical and Biophysical Research Communications, v.353, n.4, p. 849-850, 2007.

http://dx.doi.org/10.1016/j.bbrc.2006.12.069

CIMMINO, A.; ANDOLFI, A.; ZONNO, M.; AVOLIO, F.; SANTINI, A.; TUZI, A.; BERESTETSKYI, A.; VURRO M.; EVIDENTE, A. Chenopodolans A-C: phytotoxic furopyrans produced by Phoma Chenopodiicola, a fungal pathogen of Chenopodium Album. Phytochemistry, v.96, n.1, p. 208-213, 2013.

http://dx.doi.org/10.1016/j.phytochem.2013.10. $\underline{007}$

COSTA, E. C.; TEIXEIRA, F. F. S.; DANTAS, T. V. M.; MELO, V. S. P.; ARAÚJO, S. A. C.; ROLIM, B. N. Princípios da estocagem e preservação de amostras microbiológicas. Ciência Animal, v.19, n.2, p. 111-122, 2009.

DHINGRA, O. D.; SINCLAIR, J. B. Long-term storage of plant pathogens. In: DHINGRA, O. D.; SINCLAIR, J. B. Basic plant pathology methods. 2. ed. Boca Raton: CRC Press, 1995. p. 61-81. https://doi.org/10.1201/9781315138138-3

EVIDENTE, M.; CIMMINO, A.; ZONNO, M. C.; MAIS, M.; BERESTETSKI, A.; SANTORO, E.; SUPERCHI, S.; VURRO, M.; EVIDENTE, A. Phytotoxins produced by Phoma Chenopodiicola, a fungal pathogen of Chenopodium Album. Phytochemistry, v.117, n.1, p. 482-488, 2015. https://doi.org/10.1016/j.phytochem.2015.07.00 $\underline{8}$

FERREIRA, D. F. Sisvar: a guide for its bootstrap procedures in multiple comparisons. Ciência \& Agrotecnologia, v.38, n.2, p. 109-112, 2014. http://dx.doi.org/10.1590/S141370542014000200001

FINATTI, D.; APARECIDO, C. C. Caracterização fisiológica e comparação de diferentes métodos na preservação, em laboratório, de isolados do gênero Verticillium. Arquivos do Instituto Biológico, v.76, n.4, p. 715-720, 2009. 
FONG, Y. K.; ANUAR, S.; LIM, H. P.; THAM, F. Y.; SANDERSON, F. R. A modified filter paper technique for long-term preservation of some fungal cultures. Mycologist, v.4, n.3, p. 127-130, 2000.

https://doi.org/10.1016/S0269915X(00)80090-7

GIRÃO, M. D. PRADO, M. R.; BRILHANTE, R. S. N.; CORDEIRO R. A.; MONTEIRO A. J.; SIDRIM, J. J. C.; ROCHA, M. F. G. Viabilidade de cepas de Malassezia Pachydermatis mantidas em diferentes métodos de conservação. Revista da Sociedade Brasileira de Medicina Tropical, v.37, n.3, p. 229-233, 2004.

http://dx.doi.org/10.1590/S0037-

$\underline{86822004000300007}$

GREEN, L. H. Practical handbook of microbiology. 2. ed. London: CRC, 2008.

GUIMARÃES, L. C. Métodos de preservação de fungos potencialmente toxicogênicos. 2011. $55 \mathrm{f}$. Dissertação (Mestrado em Ciência dos Alimentos) - Universidade Federal de Lavras, Minas Gerais, 2011.

HINZ, H. L.; SCHWARZLÄNDER, M.; GASSMANN, A.; BOURCHIER, R. S. Successes we may not have had: a retrospective analysis of selected weed biological control agents in the United States. Invasive Plant Science and Management, v.7, n.4, p. 565-579, 2014.

https://doi.org/10.1614/IPSM-D-13-00095.1

HUSSAIN, H.; KOCK, I.; AL-HARRASI, A.; ALRAWAHI, A.; ABBAS, G.; GREEN, I. R.; SHAH, A.; BADSHAH, A.; SALEEM, M.; DRAEGER, S.; SCHULZ, B.; KROHN, K. Antimicrobial chemical constituents from endophytic fungus Phoma sp. Asian Pacific Journal of Tropical Medicine, v.7, n.9, p. 699-702, 2014.

https://doi.org/10.1016/S1995-7645(14)60119-X

KIM, E. L.; LI, J. L.; DANG, H. T.; HONG, J.; LEE, C. O.; KIM, D. K.; YOON, W. D.; KIM, E.; LIU, Y.; JUNG, J. H. Cytotoxic cytochalasins from the endozoic fungus Phoma sp. of the giant jellyfish Nemopilema Nomurai. Bioorganic \& Medicinal Chemistry Letters, v.22, n.9, p. 5752-5752, 2012. http://dx.doi.org/10.1016/i.bmcl.2012.03.058

KLAIC, R.; SALLET, D.; FOLETTO, E. L.; JACQUES, R. J. S.; GUEDES, J. V. C.; KUHN, R. C.; MAZUTTI, M.
A. Optimization of solid-state fermentation for bioherbicide production by Phoma sp. Brazilian Journal of Chemical Engineering, v.34, n.02, p. 377-384, 2017. http://dx.doi.org/10.1590/0104$\underline{6632.20170342 s 20150613}$

KOYAMA, N.; NAGAHIRO, T.; YAMAGUCHI, Y.; OHSHIRO, T.; MASUMA, R.; TOMODA, H.; OMURA, S. Spylidone, a novel inhibitor of lipid droplet accumulation in mouse macrophages produced by Phoma sp. FKI-1840. Journal of Antibiotics, v.58, n.5, p. 338-345, 2005.

http://dx.doi.org/10.1038/ia.2005.42

MAIA, F. G. M.; ARMESTO, C.; ZANCAN, W. L. A.; MAIA, J. B.; ABREU, M. S. Efeito da temperatura no crescimento micelial, produção e germinação de conídios de Colletotrichum spp. isolados de mangueira com sintomas de antracnose. Bioscience Journal, v. 27, n. 2, p. 205-210, 2011.

NAKASONE, K. K.; PETERSON, A. W.; JONG, S. Preservation and distribution of fungal cultures. In: MUELLER, G. M.; BILLS, G. F.; FOSTER, M. S. Biodiversity of fungi, inventory and monitoring methods. San Diego: Elsevier Academic Press, 2004. p. 37-47. https://doi.org/10.1016/B978012509551-8/50006-4

PASSADOR, M. M.; PIRES, G. C. C.; FINATTI, D.; APARECIDO, C. C.; FIGUEIREDO, M. B. Manutenção da viabilidade e patogenicidade de culturas mantidas na micoteca "Mário Barreto Figueiredo". Biológico, v.72, n.1, p. 51-55, 2010.

PIRES, G. C. C.; APARECIDO, C. C.; FINATTI, D. Preservação em laboratório de fungos filamentosos por longos períodos de tempo. Biológico, v.47, n.1, p. 9-16, 2012.

QIANGQIANG, Z.; JIAJUN, W; LI, L. Storage of fungi using sterile distilled water orlyophilization: comparison after 12 years. Mycoses, v.41, n.5-6, p. 255-257, 1998.

http://dx.doi.org/10.1111/j.1439-

0507.1998.tb00334.x

RAI, M. DESHMUKH, P.; GADE, A.; INGLE, A.; KÖVICS, G. J.; IRINYI, L. Phoma Saccardo: distribution, secondary metabolite production and biotechnological applications. Critical Reviews in Microbiology, v.35, n.3, p. 182-196, 2009.

http://dx.doi.org/10.1080/10408410902975992 
SANTIAGO, C.; CHRIS FITCHETT, C.; MUNRO, M. H. G.; JALIL, J.; SANTHANAM, J. Cytotoxic and antifungal activities of 5-Hydroxyramulosin, a compound produced by an endophytic fungus isolated from Cinnamomum Mollisimum. Evidence-Based Complementary and Alternative Medicine, v.12, n.10, p. 1-6, 2012. http://dx.doi.org/10.1155/2012/689310

SOLA, M. C.; OLIVEIRA, A. P.; FEISTEL, J. C.; REZENDE, C. S. M. Manutenção de microrganismos: conservação e viabilidade. Enciclopédia Biosfera, v.8, n.14, p. 1398-1418, 2012.

TERAMOTO, A.; MARTINS, M. C.; CUNHA, M. G. Avaliação de métodos para preservação de isolados de Corynespora Cassiicola (Berk. \& M.A. Curtis) C.T. Wei. Pesquisa Agropecuária Tropical, v.41, n.2, p. 296-298, 2011.

https://doi.org/10.5216/pat.v41i2.12571

TODERO, I.; CONFORTIN, T. C.; LUFT, L.; BRUN, T.; UGALDE, G. A.; ALMEITA, T. C.; ARNEMANN, J. A.; ZABOT, G. L.; MAZUTTI, M. A. Formulation of a bioherbicide with metabolites from Phoma sp. Scientia Horticulturae, v.241, n.1, p. 285-292, 2018a.

https://doi.org/10.1016/i.scienta.2018.07.009

TODERO, I.; CONFORTIN, T. C.; SOARES, J. F.; BRUN, T.; LUFT, L.; RABUSKE, J. E.; KUHN, R. C.; TRES, M. V.; ZABOT, G. L.; MAZUTTI, M. A. Concentration of metabolites from Phoma sp. using microfiltration membrane for increasing bioherbicidal activity. Environmental Technology, v.22, n.1, p. 1-9. 2018 b.

https://doi.org/10.1080/09593330.2018.1441330

VILLAVERDE, J. J.; MORÁN, B. S.; SANDÍN-ESPAÑA, P.; ALONSO-PRADOS J. L.; LÓPEZ-GOTI, C. Biopesticides in the framework of the european pesticide regulation (EC) No. 1107/2009. Pest Management Science, v.70, n.1, p. 2-5, 2014. https://doi.org/10.1002/ps.3663

WIJERATNE, E. M. K.; HE, H.; FRANZBLAU, S. G.; HOFFMAN, A. M.; GUNATILAKA, A. A. L. Phomapyrrolidones A-C, Antitubercular Alkaloids from the Endophytic Fungus Phoma sp. NRRL 46751. Journal of Natural Products, v.76, n.10, p. 1860-1865, 2013.

http://dx.doi.org/10.1021/np400391p
YANO, T.; AOYAGI, A.; KOZUMA, S.; KAWAMURA, Y.; TANAKA, I.; SUZUKI, Y.; TAKAMATSU, Y.; TAKATSU, T.; INUKAI, M. Pleofungins, novel inositol phosphorylceramide synthase inhibitors from Phoma sp. SANK 13899. Journal of Antibiotics, v.60, n.2, p. 136-142, 2007. http://dx.doi.org/10.1038/ia.2007.13 